Article

\title{
Low Dimensional Vessiot-Guldberg-Lie Algebras of Second-Order Ordinary Differential Equations
}

\author{
Rutwig Campoamor-Stursberg \\ Instituto de Matemática Interdisciplinar and Depto. Geometría y Topología, Universidad Complutense de \\ Madrid, Plaza de Ciencias 3, Madrid E-28040, Spain; rutwig@ucm.es; Tel.: +34-913944571 \\ Academic Editor: Roman M. Cherniha \\ Received: 1 December 2015 ; Accepted: 26 February 2016 ; Published: 17 March 2016
}

\begin{abstract}
A direct approach to non-linear second-order ordinary differential equations admitting a superposition principle is developed by means of Vessiot-Guldberg-Lie algebras of a dimension not exceeding three. This procedure allows us to describe generic types of second-order ordinary differential equations subjected to some constraints and admitting a given Lie algebra as Vessiot-Guldberg-Lie algebra. In particular, well-known types, such as the Milne-Pinney or Kummer-Schwarz equations, are recovered as special cases of this classification. The analogous problem for systems of second-order differential equations in the real plane is considered for a special case that enlarges the generalized Ermakov systems.
\end{abstract}

Keywords: Lie systems; Vessiot-Guldberg-Lie algebra; superposition rule; SODE Lie systems

\section{Introduction}

The theory of Lie systems, i.e., systems of non-autonomous first-order ordinary differential equations admitting a (generally nonlinear) superposition principle, is an old one and emerges principally from the pioneering work of Lie, Vessiot and Guldberg in the late 19th century [1]. For a long time considered as a particular technique for differential equations, the importance of Lie systems in physical applications, particularly in the context of integrable systems, as well as in control theory, has motivated extensive studies on the subject in the last few decades (see, e.g., [2,3]) that have led to natural generalizations of the notion of Lie systems [4-6]. These generalizations of the classical analytical formulation allow an elegant and effective description of Lie systems in terms of distributions in the sense of Fröbenius and the theory of fiber bundles, hence offering a much wider spectrum of applications, as well as their adaptation to quantum systems (the excellent treatise on the geometrical foundations of Lie systems [2] contains an extensive and updated list of references enumerating these applications).

In this work, we reconsider, mainly for the case of scalar second-order ordinary differential equations (scalar SODE Lie systems), a direct approach to Vessiot-Guldberg-Lie algebras in dimensions not exceeding three. Based on the particularities of the case of two-dimensional Vessiot-Guldberg-Lie algebras, the admissible realizations in terms of vector fields for three dimensions are obtained, enabling a direct construction of differential equations associated with these algebras.

We stress the fact that the multiple results concerning Lie algebras of vector fields could be used (see, e.g., [7]) and that the superposition problem has been studied from this perspective by various authors [8-10]. The purpose of our direct construction is of a practical nature, in order to illustrate that cleverly-chosen constraints on realizations allow the construction of quite general differential equations (systems) admitting Vessiot-Guldberg-Lie algebras. 
Definition: A system of ordinary differential equations:

$$
\frac{d x^{i}}{d t}=\Psi^{i}(t, \mathbf{x}), \quad 1 \leq i \leq n
$$

is said to admit a fundamental system of solutions if the general solution of the system can be expressed in terms of a finite number $m$ of functionally independent particular solutions $\left\{\mathbf{y}_{1}, \cdots, \mathbf{y}_{m}\right\}$ and $n$ significant constants $\left\{C_{1}, \cdots, C_{n}\right\}$ :

$$
x^{i}=\varphi^{i}\left(\mathbf{y}_{1}, \cdots, \mathbf{y}_{m}, C_{1}, \cdots, C_{n}\right)
$$

where $\mathbf{y}_{k}=\left(y_{k}^{1}, \cdots, y_{k}^{n}\right)$. We say that Equation (2) is a superposition rule for the system Equation (1), while the set $\left\{\mathbf{y}_{1}, \cdots, \mathbf{y}_{m}\right\}$ is called a fundamental system of solutions. The vector field in $\mathbb{R}^{n}$ defined by:

$$
\mathbf{X}(t, \mathbf{x})=\sum_{i=1}^{n} \Psi^{i}(t, \mathbf{x}) \frac{\partial}{\partial x^{i}}
$$

is further called the $t$-dependent vector field associated with the system Equation (1). It is immediate to verify that there is a one-to-one correspondence between systems and vector fields of this type.

The basic criterion concerning the existence of fundamental systems of solutions is due to Lie himself [1] and can be formulated as follows [2,11]:

Lie-Scheffers Theorem [1]: The system of ODEs Equation (1) possesses a generic fundamental system of solutions if it can be represented in the form:

$$
\frac{d x^{i}}{d t}=\sum_{k=1}^{r} F_{k}(t) \xi_{k}^{i}(\mathbf{x})
$$

such that the vector fields:

$$
X_{\alpha}=\sum_{i=1}^{n} \xi_{\alpha}^{i}(\mathbf{x}) \frac{\partial}{\partial x^{i}}, 1 \leq \alpha \leq r
$$

span an $r$-dimensional Lie algebra $\mathcal{L}_{V G}$. Moreover, the so-called Lie-condition:

$$
n m \geq r=\operatorname{dim} \mathcal{L}_{V G}
$$

is satisfied.

This criterion implies in particular that the $t$-dependent vector field Equation (3) can be written as:

$$
\mathbf{X}(t, x, v)=\sum_{\alpha=1}^{r} F_{\alpha}(t) X_{\alpha}
$$

The Lie algebra $\mathcal{L}_{V G}$ is usually called a Vessiot-Guldberg-Lie algebra of the system. The existence of such an algebra hence ensures the existence of a (nonlinear) superposition principle $[2,12,13]$, which can (at least formally) be derived in terms of the differential invariants associated with the generators of a Vessiot-Guldberg-Lie algebra.

It shall however be observed that, in contrast to the Lie algebra of point symmetries of differential equations [11,12], a Vessiot-Guldberg-Lie algebra generally does not provide an invariant of the system. Actually, the same system Equation (1) may admit different superposition rules, hence non-isomorphic Vessiot-Guldberg-Lie algebras that can have different dimensions. The Riccati equation and other ODEs related to it constitute the canonical examples for this pathology [2]. In this sense, the problem differs considerably from the construction of differential equations admitting certain types of symmetry algebras [14]. 


\section{Vessiot-Guldberg-Lie Algebras with $r \leq 3$ for Scalar SODE Systems}

In this section, realizations of Vessiot-Guldberg-Lie algebras in low dimensions are analyzed directly for the case of a scalar second-order ODE, corresponding formally to the case of first-order systems with $n=2$.

A scalar second-order ODE:

$$
\ddot{x}-F(t, x, \dot{x})=0
$$

can always be rewritten as a first-order system:

$$
\begin{aligned}
& \dot{x}=v \\
& \dot{v}=F(t, x, v)
\end{aligned}
$$

with $n=2$. In this case, the $t$-dependent vector field $\mathbf{X}(t, x, v)$ has the form:

$$
\mathbf{X}(t, x, v)=v \frac{\partial}{\partial x}+F(t, x, v) \frac{\partial}{\partial v}
$$

We say that Equation (7) is a SODE Lie system if it possesses a Vessiot-Guldberg-Lie algebra $\mathcal{L}_{V G}$ Geometrically, the vector field $\mathbf{X}(t, x, v)$ takes values in the Lie algebra $\mathcal{L}_{V G}$; hence, in particular, for two given values $t_{0} \neq t_{1}$, the vector fields:

$$
\mathbf{X}\left(t_{0}, x, v\right)=v \frac{\partial}{\partial x}+F\left(t_{0}, x, v\right) \frac{\partial}{\partial v}, \mathbf{X}\left(t_{0}, x, v\right)-\mathbf{X}\left(t_{1}, x, v\right)=\left(F\left(t_{0}, x, v\right)-F\left(t_{1}, x, v\right)\right) \frac{\partial}{\partial v}
$$

must be elements of $\mathcal{L}_{V G}$. Considering the determinant of the matrix of coefficients of these vector fields, we find that:

$$
\left|\begin{array}{cc}
v & F\left(t_{0}, x, v\right) \\
0 & F\left(t_{0}, x, v\right)-F\left(t_{1}, x, v\right)
\end{array}\right|=v\left(F\left(t_{0}, x, v\right)-F\left(t_{1}, x, v\right)\right)
$$

Now, if $\operatorname{dim} \mathcal{L}_{V G}=1$, then Equation (10) must vanish for all values of $t$, from which we conclude that:

$$
F\left(t_{0}, x, v\right)=F(t, x, v)
$$

for all $t \in \mathbb{R}$, implying that the constraint $\frac{\partial F}{\partial t}=0$ must be satisfied. The SODE Lie system thus reduces to:

$$
\ddot{x}-F(x, \dot{x})=0
$$

and the generator of $\mathcal{L}_{V G}$ is given by $X_{1}=v \frac{\partial}{\partial x}+F(x, v) \frac{\partial}{\partial v}$.

The substitution $\dot{x}=p$ further reduces Equation (11) to a first-order equation, recovering the well-known property that autonomous second-order ODEs admit a superposition principle. The same conclusion holds for autonomous SODE Lie systems in higher dimensions $[5,13]$.

\section{1. $\operatorname{dim} \mathcal{L}_{V G}=2$}

The simplest case of a non-trivial Vessiot-Guldberg-Lie algebra is given by a Lie algebra in dimension two, as it is either Abelian or isomorphic to the affine algebra $\mathfrak{a}_{2}$ on the plane [15] (in the following, the reader is led to this reference for the elementary properties of Lie algebras; for classifications in low dimensions, see $[15,16])$. Suppose thus that the system Equation (8) admits a Vessiot-Guldberg-Lie algebra $\mathcal{L}_{V G}$ of dimension two (it is implicitly assumed that it does not admit a smaller dimensional Lie algebra $\mathcal{L}_{V G}$ ). By Equation (6), we have the identity:

$$
\mathbf{X}(t, x, v)=v \frac{\partial}{\partial x}+F(t, x, v) \frac{\partial}{\partial v}=F_{1}(t) X_{1}+F_{2}(t) X_{2}
$$


where $X_{1}, X_{2}$ are two independent elements of $\mathcal{L}_{V G}$. Using again the vector fields Equation (9), there exist $t_{0} \neq t_{1}$, such that the determinant Equation (10) does not vanish:

$$
v\left(F\left(t_{0}, x, v\right)-F\left(t_{1}, x, v\right)\right) \neq 0
$$

showing that the vector fields are linearly independent. Without loss of generality, we can define a basis of $\mathcal{L}_{V G}$ as:

$$
X_{1}=v \frac{\partial}{\partial x}+F\left(t_{0}, x, v\right) \frac{\partial}{\partial v}, X_{2}=\left(F\left(t_{0}, x, v\right)-F\left(t_{1}, x, v\right)\right) \frac{\partial}{\partial v}
$$

We rewrite the generators of Equation (13) as:

$$
X_{1}=v \frac{\partial}{\partial x}+f(x, v) \frac{\partial}{\partial v}, X_{2}=g(x, v) \frac{\partial}{\partial v}
$$

where, by Equation (12), it follows that $F_{1}(t)=1$ and $f(x, v)+F_{2}(t) g(x, v)=F(t, x, v)$ for some non-constant function $F_{2}(t)$ and $g(x, v) \neq 0$. The commutator is given by:

$$
\left[X_{1}, X_{2}\right]=-g(x, v) \frac{\partial}{\partial x}+\left(v \frac{\partial g}{\partial x}+f(x, v) \frac{\partial g}{\partial v}-g(x, v) \frac{\partial f}{\partial v}\right) \frac{\partial}{\partial v} \neq 0
$$

The only possibility that $X_{1}, X_{2}$ generate a two-dimensional Lie algebra is that $\left[X_{1}, X_{2}\right]=\lambda X_{1}+$ $\mu X_{2}$ for some $\lambda \neq 0$. This assumption leads to the equations:

$$
\begin{aligned}
g(x, v)+\lambda v & =0 \\
v \frac{\partial g}{\partial x}+f(x, v) \frac{\partial g}{\partial v}-g(x, v) \frac{\partial f}{\partial v}-\lambda f(x, v)-\mu g(x, v) & =0
\end{aligned}
$$

Solving the first of these equations and inserting the result in the second, we obtain the general solution:

$$
f(x, v)=\mu v+v^{2} f_{1}(x)
$$

A further linear change allows us to suppose that $\mu=0$. The $t$-dependent vector field is thus given by:

$$
\mathbf{X}(t, x, v)=v \frac{\partial}{\partial x}+\left(f_{1}(x) v^{2}+\lambda F_{2}(t) v\right) \frac{\partial}{\partial v}
$$

and the associated SODE Lie system is easily seen to be an equation of the Liouville type (in various applications, this type of SODE Lie system is also known as the generalized Buchdahl equation [4]):

$$
\ddot{x}-f_{1}(x) \dot{x}^{2}-\lambda F_{2}(t) \dot{x}=0
$$

This equation is well known to admit an integrating factor, a first integral being given by:

$$
\ln |\dot{x}|+\int f_{1}(x) d x+\lambda \int F_{2}(t) d t=C_{1}
$$

In this sense, a two-dimensional Vessiot-Guldberg-Lie $\mathcal{L}_{V G}$ does not provide new results to those already derived from the theory of differential equations.

\section{2. $\operatorname{dim} \mathcal{L}_{V G}=3$}

For the case of three-dimensional Vessiot-Guldberg-Lie algebras, the analysis of the generic form of the realizations of the algebra generators is quite similar to that already developed for the 
two-dimensional algebras. Suppose that Equation (7) does admit a Vessiot-Guldberg-Lie algebra with $\operatorname{dim} \mathcal{L}_{V G}=3$. By the same argument used in two dimensions, the vector fields:

$$
X_{1}=v \frac{\partial}{\partial x}+f(x, v) \frac{\partial}{\partial v}, X_{2}=g(x, v) \frac{\partial}{\partial v}
$$

are contained in $\mathcal{L}_{V G}$, where $f(x, v)=F\left(t_{0}, x, v\right)$ and $g(x, v)=F\left(t_{0}, x, v\right)-F\left(t_{1}, x, v\right)$ for some arbitrary values $t_{0} \neq t_{1}$. Further, the commutator:

$$
\left[X_{1}, X_{2}\right]=-g(x, v) \frac{\partial}{\partial x}+\left(v \frac{\partial g}{\partial x}+f(x, v) \frac{\partial g}{\partial v}-g(x, v) \frac{\partial f}{\partial v}\right) \frac{\partial}{\partial v} \neq 0
$$

also belongs to the Vessiot-Guldberg-Lie algebra $\mathcal{L}_{V G}$. The requirement on the dimension of $\mathcal{L}_{V G}$ implies that $\left[X_{1}, X_{2}\right]$ must be linearly independent on $X_{1}, X_{2}$, as otherwise $\mathcal{L}_{V G}$ reduces to the affine Lie algebra $\mathfrak{a}_{2}$. Therefore, there exists a third vector field $X_{3}=\xi_{3}^{1}(x, v) \frac{\partial}{\partial x}+\xi_{3}^{2}(x, v) \frac{\partial}{\partial v} \in \mathcal{L}_{V G}$ with $\xi_{3}^{1}(x, v) \neq 0$, such that $\left\{X_{1}, X_{2}, X_{3}\right\}$ constitute a basis of $\mathcal{L}_{V G}$ and satisfying the identity:

$$
\left[X_{1}, X_{2}\right]=\alpha X_{1}+\beta X_{2}+\gamma X_{3}
$$

for some constants $\alpha, \beta, \gamma$, where necessarily $\gamma \neq 0$.

As a consequence, a three-dimensional Vessiot-Guldberg-Lie algebra $\mathcal{L}_{V G}$ can always be realized in the following way:

$$
X_{1}=v \frac{\partial}{\partial x}+f(x, v) \frac{\partial}{\partial v} ; \quad X_{2}=g(x, v) \frac{\partial}{\partial v} ; \quad X_{3}=\xi_{3}^{1}(x, v) \frac{\partial}{\partial x}+\xi_{3}^{2}(x, v) \frac{\partial}{\partial v}
$$

In particular, the $t$-dependent vector field is given by $\mathbf{X}(t, x, v)=X_{1}+F_{2}(t) X_{2}$.

For the latter realization, the remaining commutators are given by:

$$
\begin{aligned}
& {\left[X_{1}, X_{3}\right]=\left(v \frac{\partial \xi_{3}^{1}}{\partial x}+v \frac{\partial \xi_{3}^{1}}{\partial v}-\xi_{3}^{2}(x, v)\right) \frac{\partial}{\partial x}+\left(v \frac{\partial \xi_{3}^{2}}{\partial x}+f(x, v) \frac{\partial \xi_{3}^{2}}{\partial v}-\xi_{3}^{2}(x, v)\right) \frac{\partial}{\partial v}} \\
& {\left[X_{2}, X_{3}\right]=\left(g(x, v) \frac{\partial \xi_{3}^{2}}{\partial v}-\xi_{3}^{2}(x, v) \frac{\partial g}{\partial v}\right) \frac{\partial}{\partial v}}
\end{aligned}
$$

As the component in $\frac{\partial}{\partial x}$ of $X_{2}$ is zero, a change of basis always enables us to reduce the first commutator to the form:

$$
\left[X_{1}, X_{2}\right]=\lambda X_{1}+\mu X_{3}
$$

where $\mu \neq 0$. By means of a scaling change in $X_{2}$, we can further suppose that $\mu=1$ (we observe that $\left[X_{1}, X_{2}\right]=\alpha X_{2}$ cannot appear by the previously indicated reasons). The remaining brackets have the form:

$$
\left[X_{1}, X_{3}\right]=a_{1} X_{1}+a_{2} X_{2}+a_{3} X_{3},\left[X_{2}, X_{3}\right]=b_{1} X_{1}+b_{2} X_{2}+b_{3} X_{3}
$$

for some constants $a_{i}, b_{i} \in \mathbb{R}$. If $\lambda=0$, then:

$$
\left[X_{1}, X_{2}\right]=X_{3}
$$

and a routine computation shows that the Jacobi identity is fulfilled whenever the relations $b_{2}=-a_{1}$ and:

$$
a_{1} a_{3}+a_{2} b_{3}=0, b_{1} a_{3}-a_{1} b_{3}=0
$$

are satisfied. At this stage, the problem corresponds essentially to classifying three-dimensional Lie algebras, a well-known problem solved in any standard reference [15]. We merely indicate that it suffices to consider the following three cases:
(a) $b_{3}=0, b_{1}=0, a_{1}=0$;
(b) $b_{3}=0, a_{3}=0$;
(c) $a_{3}=0, a_{1}=0, a_{2}=0$. 
We observe that in the commutators Equation (16), the role of $X_{1}$ and $X_{2}$ is symmetric, implying that Cases (a) and (c) are equivalent. For each of the remaining possibilities, the commutators Equation (16) can be further reduced by successive changes of basis. As this is a routine computation, we skip the details and will only indicate the resulting three-dimensional Lie algebras.

If $\lambda \neq 0$, a similar reasoning with the Jacobi identity and the realization Equation (15) shows that only one isomorphism class can appear, corresponding to the following values of the constants in Equation (16): $a_{1}=a_{2}=a_{3}=b_{2}=0, b_{1}=1, b_{3}=-\lambda$.

Proposition 1: If the vector fields Equation (15) generates a three-dimensional Vessiot-Guldberg-Lie algebra $\mathcal{L}_{V G}$, then it belongs to one of the following types:

1. $\mathfrak{r}_{1, \varepsilon}:$

$$
\left[X_{1}, X_{2}\right]=X_{3}, \quad\left[X_{1}, X_{3}\right]=\varepsilon X_{2}, \quad\left[X_{2}, X_{3}\right]=X_{1}, \quad \varepsilon=-1,1
$$

2. $\mathfrak{r}_{2}$ :

$$
\left[X_{1}, X_{2}\right]=X_{3}, \quad\left[X_{1}, X_{3}\right]=X_{1}, \quad\left[X_{2}, X_{3}\right]=-X_{2}
$$

3. $\mathfrak{r}_{3, \lambda}$ :

$$
\left[X_{1}, X_{2}\right]=X_{3} \quad\left[X_{1}, X_{3}\right]=0, \quad\left[X_{2}, X_{3}\right]=\lambda X_{1}, \quad \lambda \in \mathbb{R}
$$

4. $\mathfrak{r}_{4}$ :

$$
\left[X_{1}, X_{2}\right]=X_{3}, \quad\left[X_{1}, X_{3}\right]=0, \quad\left[X_{2}, X_{3}\right]=0
$$

5. $\mathfrak{r}_{5}$ :

$$
\left[X_{1}, X_{2}\right]=X_{3}, \quad\left[X_{1}, X_{3}\right]=-X_{2}, \quad\left[X_{2}, X_{3}\right]=0 .
$$

6. $\mathfrak{r}_{6, \lambda}$ :

$$
\left[X_{1}, X_{2}\right]=-\lambda X_{1}+X_{3} \quad\left[X_{1}, X_{3}\right]=0, \quad\left[X_{2}, X_{3}\right]=X_{1}+\lambda X_{3}, \quad \lambda>0 .
$$

Concerning the isomorphism class of these Lie algebras, we have that $\mathfrak{r}_{1,1}$ and $\mathfrak{r}_{2}$ are isomorphic to the simple Lie algebra $\mathfrak{s l}(2, \mathbb{R})$, while $\mathfrak{r}_{1,-1}$ is isomorphic to $\mathfrak{s o}(3)$. The remaining algebras are solvable. Following the list of [16], we have the isomorphisms $\mathfrak{r}_{3, \lambda} \simeq A_{3,8}, \mathfrak{r}_{4} \simeq A_{3,3}, \mathfrak{r}_{5} \simeq A_{3,8}$ and $\mathfrak{r}_{6, \lambda} \simeq A_{3,9}$.

Once the possible Lie algebras $\mathfrak{g}$ have been deduced, the precise functions in the realization Equation (15), as well as the differential equations admitting $\mathfrak{g}$ as Vessiot-Guldberg-Lie algebra $\mathcal{L}_{V G}$ are obtained by solving the successive conditions imposed by the commutators of $\mathfrak{g}$. As an illustration of the procedure, we give the details for the Lie algebras $\mathfrak{r}_{1, \varepsilon}$, the remaining cases being obtained in a completely analogous way.

Let $\mathcal{L}_{V G}$ have brackets (18) with $\varepsilon= \pm 1$. From the two first generators:

$$
X_{1}=v \frac{\partial}{\partial x}+f(x, v) \frac{\partial}{\partial v}, X_{2}=g(x, v) \frac{\partial}{\partial v}
$$

it follows that the third generator has the form:

$$
X_{3}:=\left[X_{1}, X_{2}\right]=-g(x, v) \frac{\partial}{\partial x}+\left(v \frac{\partial g}{\partial x}+f(x, v) \frac{\partial g}{\partial v}-g(x, v) \frac{\partial f}{\partial v}\right) \frac{\partial}{\partial v}
$$


In principle, four conditions on $f(x, v)$ and $g(x, v)$ corresponding to the components of the remaining commutators $\left[X_{1}, X_{3}\right]$ and $\left[X_{2}, X_{3}\right]$ must be solved. These are explicitly given by:

$$
\begin{aligned}
{\left[X_{1}, X_{3}\right]=} & \left(g(x, v) \frac{\partial f}{\partial v}-2 v \frac{\partial g}{\partial x}-2 f(x, v) \frac{\partial g}{\partial v}\right) \frac{\partial}{\partial x}+g(x, v)\left(\frac{\partial f}{\partial x}-\varepsilon+\left(\frac{\partial f}{\partial v}\right)^{2}\right) \frac{\partial}{\partial v} \\
& +f(x, v)\left(\frac{\partial g}{\partial x}-g(x, v) \frac{\partial^{2} f}{\partial v^{2}}-\frac{\partial f}{\partial v} \frac{\partial g}{\partial v}+2 v \frac{\partial^{2} g}{\partial x \partial v}+f(x, v) \frac{\partial^{2} g}{\partial v^{2}}\right) \frac{\partial}{\partial v} \\
& +v\left(\frac{\partial f}{\partial x} \frac{\partial g}{\partial v}-2 \frac{\partial f}{\partial v} \frac{\partial g}{\partial x}+v \frac{\partial^{2} g}{\partial x^{2}}-g(x, v) \frac{\partial^{2} f}{\partial x \partial v}\right) \frac{\partial}{\partial v} \\
{\left[X_{2}, X_{3}\right]=} & -g(x, v) \frac{\partial g}{\partial v} \frac{\partial}{\partial x}+g(x, v)\left(\frac{\partial f}{\partial v} \frac{\partial g}{\partial v}+2 \frac{\partial g}{\partial x}+v \frac{\partial^{2} g}{\partial x \partial v}+f(x, v) \frac{\partial^{2} g}{\partial v^{2}}\right) \frac{\partial}{\partial v} \\
& -\left(g(x, v)^{2} \frac{\partial^{2} f}{\partial v^{2}}+f(x, v)\left(\frac{\partial g}{\partial v}\right)^{2}-v \frac{\partial g}{\partial v} \frac{\partial g}{\partial x}\right) \frac{\partial}{\partial v}
\end{aligned}
$$

As $\left[X_{2}, X_{3}\right]=X_{1}$ holds, from the component in $\frac{\partial}{\partial x}$, we extract the first-order PDE:

$$
-g(x, v) \frac{\partial g}{\partial v}=v
$$

with generic solution:

$$
g(x, v)=\sqrt{G(x)-v^{2}}
$$

for some function $G(x)$. Inserting $g(x, v)$ into the commutators, the component in $\frac{\partial}{\partial x}$ of the commutator $\left[X_{1}, X_{3}\right]$ further leads to the condition:

$$
v \frac{d G}{d x}-2 v f(x, v)+v^{2} \frac{\partial f}{\partial v}-G(x) \frac{\partial f}{\partial v}=0
$$

The latter PDE has general solution:

$$
f(x, v)=\left(G(x)-v^{2}\right) k(x)+\frac{1}{2} \frac{d h}{d x}
$$

It turns out that with this choice of $f(x, v)$, the commutator $\left[X_{2}, X_{3}\right]=X_{1}$ is identically satisfied. It merely remains to consider the condition specified by the component in $\frac{\partial}{\partial v}$ of $\left[X_{1}, X_{3}\right]$, given by:

$$
\frac{d^{2} G}{d x^{2}}-3 k(x) \frac{d G}{d x}-2 G(x) \frac{d k}{d x}+2 G(x) k^{2}(x)-2 \varepsilon=0
$$

This constraint equation shows that the realization Equations (24) and (25) depends on an arbitrary function $G(x)$. For any pair of functions $\{G(x), k(x)\}$ satisfying Equation (26), the commutators Equation (18) is satisfied and defines a three-dimensional Vessiot-Guldberg-Lie algebra for the (nonlinear) second-order ODE:

$$
\ddot{x}=k(x) \dot{x}^{2}+F_{2}(t) \sqrt{G(x)-\dot{x}^{2}}+\frac{1}{2} \frac{d G}{d x}-G(x) k(x)=0
$$

Recall that for $\varepsilon=1$, the Vessiot-Guldberg-Lie algebra is isomorphic to $\mathfrak{s l}(2, \mathbb{R})$, while for $\varepsilon=-1$, it is isomorphic to $\mathfrak{s o}(3)$.

\subsection{Second-Order ODEs with Three-Dimensional Vessiot-Guldberg-Lie Algebra}

For each of the Lie algebras $\mathfrak{g}$ listed in Proposition 1, we enumerate the realization by vector fields, the possible constraints on the functions in their components and the generic SODE Lie system admitting $\mathfrak{g}$ as a Vessiot-Guldberg Lie algebra.

1. $\mathcal{L}_{V G} \simeq \mathfrak{r}_{1, \varepsilon}:$

Commutators:

$$
\left[X_{1}, X_{2}\right]=X_{3}, \quad\left[X_{1}, X_{3}\right]=\varepsilon X_{2}, \quad\left[X_{2}, X_{3}\right]=X_{1}
$$


Realization:

$$
\begin{aligned}
& X_{1}=v \frac{\partial}{\partial x}+\left(\frac{G^{\prime}(x)}{2}-k(x)\left(G(x)-v^{2}\right)\right) \frac{\partial}{\partial v}, \quad X_{2}=\sqrt{G(x)-v^{2}} \frac{\partial}{\partial v} \\
& X_{3}=-\sqrt{G(x)-v^{2}}\left(\frac{\partial}{\partial x}+v k(x) \frac{\partial}{\partial v}\right)
\end{aligned}
$$

Second-order differential equation:

$$
\ddot{x}=k(x) \dot{x}^{2}+F_{2}(t) \sqrt{G(x)-\dot{x}^{2}}+\frac{1}{2} G^{\prime}(x)-k(x) G(x)
$$

Constraints:

$$
G^{\prime \prime}(x)-2 k^{\prime}(x) G(x)+2 k^{2}(x) G(x)-3 k(x) G^{\prime}(x)-2 \varepsilon=0
$$

2. $\mathcal{L}_{V G} \simeq \mathfrak{r}_{2}$ :

Commutators:

$$
\left[X_{1}, X_{2}\right]=X_{3}, \quad\left[X_{1}, X_{3}\right]=X_{1}, \quad\left[X_{2}, X_{3}\right]=-X_{2}
$$

Realization:

$$
X_{1}=v \frac{\partial}{\partial x}+\left(k(x)+\frac{\left(1+2 G^{\prime}(x)\right)}{2 G(x)} v^{2}\right) \frac{\partial}{\partial v}, \quad X_{2}=G(x) \frac{\partial}{\partial v}, \quad X_{3}=-G(x) \frac{\partial}{\partial x}-v\left(G^{\prime}(x)+1\right) \frac{\partial}{\partial v}
$$

Second-order differential equation:

$$
\ddot{x}=\frac{\dot{x}^{2}}{2}\left(\frac{1+2 G^{\prime}(x)}{G(x)}\right)+k(x)+F_{2}(t) G(x)
$$

Constraints:

$$
k^{\prime}(x) G(x)-k(x) G^{\prime}(x)-2 k(x)=0
$$

3. $\mathcal{L}_{V G} \simeq \mathfrak{r}_{3, \lambda}$ :

Commutators:

$$
\left[X_{1}, X_{2}\right]=X_{3}, \quad\left[X_{1}, X_{3}\right]=0, \quad\left[X_{2}, X_{3}\right]=\lambda X_{1}
$$

Realization:

$$
\begin{aligned}
& X_{1}=v \frac{\partial}{\partial x}+\left(k(x)\left(G(x)-\lambda v^{2}\right)+\frac{G^{\prime}(x)}{2 \lambda}\right) \frac{\partial}{\partial v}, \quad X_{2}=\sqrt{G(x)-\lambda v^{2}} \frac{\partial}{\partial v}, \\
& X_{3}=-\sqrt{G(x)-\lambda v^{2}}\left(\frac{\partial}{\partial x}-\lambda v k(x)\right) \frac{\partial}{\partial v}
\end{aligned}
$$

Second-order differential equation:

$$
\ddot{x}=-\lambda k(x) \dot{x}^{2}+G(x) k(x)+\frac{G^{\prime}(x)}{2 \lambda}+F_{2}(t) \sqrt{G(x)-\lambda \dot{x}^{2}}
$$

Constraints:

$$
2 \lambda^{2} G^{\prime}(x) k^{2}(x)+2 \lambda G(x) k^{\prime}(x)+3 \lambda G(x) k(x)+G^{\prime \prime}(x)=0
$$

4. $\mathcal{L}_{V G} \simeq \mathfrak{r}_{4}:$

Commutators:

$$
\left[X_{1}, X_{2}\right]=X_{3}, \quad\left[X_{1}, X_{3}\right]=0, \quad\left[X_{2}, X_{3}\right]=0
$$

Realization:

$$
X_{1}=v \frac{\partial}{\partial x}+\frac{G^{\prime}(x) v^{2}+\mu G^{2}(x)}{G(x)} \frac{\partial}{\partial v}, \quad X_{2}=G(x) \frac{\partial}{\partial v}, \quad X_{3}=-G(x) \frac{\partial}{\partial x}-v G^{\prime}(x) \frac{\partial}{\partial v}
$$


Second-order differential equation:

$$
\ddot{x}=\frac{G^{\prime}(x)}{G(x)} \dot{x}^{2}+\left(F_{2}(t)+\mu\right) G(x)
$$

5. $\mathcal{L}_{V G} \simeq \mathfrak{r}_{5}$ :

Commutators:

$$
\left[X_{1}, X_{2}\right]=X_{3}, \quad\left[X_{1}, X_{3}\right]=-X_{2}, \quad\left[X_{2}, X_{3}\right]=0
$$

Realization:

$$
X_{1}=v \frac{\partial}{\partial x}+\left(\frac{G^{\prime}(x)}{G(x)} v^{2}+k(x)\right) \frac{\partial}{\partial v}, \quad X_{2}=G(x) \frac{\partial}{\partial v}, \quad X_{3}=-G(x) \frac{\partial}{\partial x}-v G^{\prime}(x) \frac{\partial}{\partial v}
$$

Second-order differential equation:

$$
\ddot{x}=\frac{G^{\prime}(x)}{G(x)} \dot{x}^{2}+k(x)+F_{2}(t) G(x)
$$

Constraints:

$$
k^{\prime}(x) G(x)-k(x) G^{\prime}(x)+G(x)=0
$$

6. $\mathcal{L}_{V G} \simeq \mathfrak{r}_{6, \lambda}$ :

Commutators:

$$
\left[X_{1}, X_{2}\right]=-\lambda X_{1}+X_{3}, \quad\left[X_{1}, X_{3}\right]=0, \quad\left[X_{2}, X_{3}\right]=X_{1}+\lambda X_{3}
$$

Realization:

$$
X_{1}=v \frac{\partial}{\partial x}+\frac{G^{2}(v)}{\left(\beta+\left(1+\lambda^{2}\right) x\right)} \frac{\partial}{\partial v}, \quad X_{2}=G(v) \frac{\partial}{\partial v}, \quad X_{3}=(\lambda v-G(v)) \frac{\partial}{\partial x}-\frac{G^{2}(v)\left(G^{\prime}(v)-\lambda\right)}{\left(\beta+\left(1+\lambda^{2}\right) x\right)} \frac{\partial}{\partial v}
$$

Second-order differential equation:

$$
\ddot{x}=\frac{G^{2}(\dot{x})}{\left(\beta+\left(1+\lambda^{2}\right) x\right)}+F_{2}(t) G(\dot{x})
$$

Constraints:

$$
2 \lambda G(v)-G(v) G^{\prime}(v)-\left(1+\lambda^{2}\right) v=0
$$

We observe that, in most cases, the preceding SODE Lie systems always involve a term quadratic in the velocity, with the exception of Equation (36), where more general functions of $\dot{x}$ are allowed.

\subsection{Examples}

The preceding generic realizations of Vessiot-Guldberg-Lie algebras must in particular cover the already known SODE Lie systems for which the condition $\operatorname{dim} \mathcal{L}_{V G} \leq 3$ holds.

Among the low dimensional Lie algebras, the case $\mathfrak{r}_{2} \simeq \mathfrak{s l}(2, \mathbb{R})$ plays a special role, as it is related to various of the most relevant and best studied cases of SODE Lie systems (see, e.g., [2,4,5] and the references therein).

1. The well-known Milne-Pinney equation:

$$
\ddot{x}=F(t) x+\frac{c}{x^{3}}, c \in \mathbb{R}
$$


appears making the choice $G(x)=-\frac{1}{2} x$ in Equation (29). Here, the constraint Equation (30) is given by:

$$
-\frac{x}{2} k^{\prime}(x)-\frac{3}{2} k(x)=0
$$

The solution of this equation is immediate and equals $k(x)=c x^{-3}$. Now, setting $F_{2}(t)=$ $-2 F(t)$, we recover Equation (38). In particular, for $c=0$ and $F(t)=\omega^{2}(t)$, we get the harmonic oscillator with time-dependent frequency. The Lie algebra $\mathcal{L}_{V G}$ is realized by the vector fields:

$$
X_{1}=v \frac{\partial}{\partial x}+\frac{c}{x^{3}} \frac{\partial}{\partial v}, X_{2}=-\frac{x}{2} \frac{\partial}{\partial v}, X_{3}=-\frac{x}{2} \frac{\partial}{\partial x}-\frac{v}{2} \frac{\partial}{\partial v}
$$

We observe that the Milne-Pinney equation is the only SODE Lie system with Vessiot-Guldberg-Lie algebra $\mathfrak{r}_{2}$, such that the differential equation does not contain a factor in $\dot{x}^{2}$.

2. The Kummer-Schwarz equation:

$$
\ddot{x}=\frac{3}{2 x} \dot{x}^{2}-2 c x^{3}+2 g(t) x
$$

is also recovered from Equation (29). In this case, we take $G(x)=x$, leading to the constraint Equation (30):

$$
x k^{\prime}(x)-3 k(x)=0
$$

The solution is given by $k(x)=c x^{3}$. The vector fields realizing the Lie algebra $\mathfrak{r}_{2}$ are:

$$
X_{1}=v \frac{\partial}{\partial x}+\left(c x^{3}+\frac{3}{2 x}\right) \frac{\partial}{\partial v}, X_{2}=x \frac{\partial}{\partial v}, X_{3}=-x \frac{\partial}{\partial x}-2 v \frac{\partial}{\partial v}
$$

We observe that both equations actually arise as particular cases for the value $a=0$ of the more general SODE Lie system:

$$
\ddot{x}=\frac{\left(3+m+2 a m(m-1) x^{m-1}\right)}{2\left(2+a(m-1) x^{m-1}\right)} \dot{x}^{2}+\frac{\left(2+a(m-1) x^{m-1}\right)}{(m-1)} F_{2}(t)+b x^{m}
$$

where $a, b, m \in \mathbb{R}$ and $m \neq 1$.

\section{SODE Lie Systems in the Plane}

The analogous problem of determining the systems of second-order ordinary differential equations:

$$
\ddot{x}_{1}=F_{1}\left(t, x_{1}, x_{2}, \dot{x}_{1}, x_{2}\right), \quad \ddot{x}_{2}=F_{2}\left(t, x_{1}, x_{2}, \dot{x}_{1}, x_{2}\right)
$$

in the plane admitting a Vessiot-Guldberg-Lie algebra $\mathcal{L}_{V G}$ is considerably more complicated, as the algebras are realized in $\mathbb{R}^{4}$ with the coordinates $\left\{x_{1}, x_{2}, v_{1}=\dot{x}_{1}, v_{2}=\dot{x}_{2}\right\}$, and the constraints determined by the commutators lead to (nonlinear) partial differential equations that can only seldom be solved in full generality. In this sense, a direct approach as developed for scalar equations is not practical, unless some simplifications in the realizations are introduced.

Using the straightforward generalization of the realization Equation (14), it can be easily shown that the most general SODE Lie system admitting a two-dimensional Vessiot-Guldberg-Lie algebra $\mathcal{L}_{V G}$ has the generic form:

$$
\ddot{x}_{1}-F\left(x_{1}, x_{2}, \frac{\dot{x}_{2}}{\dot{x}_{1}}\right) \dot{x}_{1}^{2}+\lambda F_{2}(t) \dot{x}_{1}=0, \quad \ddot{x}_{2}-G\left(x_{1}, x_{2}, \frac{\dot{x}_{2}}{\dot{x}_{1}}\right) \dot{x}_{1}^{2}+\lambda F_{2}(t) \dot{x}_{2}=0
$$


where we specifically have $X_{1}=v_{1} \frac{\partial}{\partial x_{1}}+v_{2} \frac{\partial}{\partial x_{2}}+F\left(x_{1}, x_{2}, \frac{\dot{x}_{2}}{\dot{x}_{1}}\right) \dot{x}_{1}^{2} \frac{\partial}{\partial v_{1}}+G\left(x_{1}, x_{2}, \dot{x}_{2}\right) \dot{x}_{1}^{2} \frac{\partial}{\partial x_{2}}$ and $X_{2}=-\lambda\left(v_{1} \frac{\partial}{\partial v_{1}}+v_{2} \frac{\partial}{\partial v_{2}}\right)$ with commutator $\left[X_{1}, X_{2}\right]=\lambda X_{1}$ (for $\lambda=0$, the system is autonomous and clearly admits a superposition principle [2]).

This result suggests an ansatz based on realizations of the type Equations (18)-(23) and such that the components of the vector field $X_{2}$ are linear functions in the coordinates $\left\{x_{1}, x_{2}, v_{1}, v_{2}\right\}$. We will only consider one case, namely that corresponding to the special case:

$$
X_{2}=\lambda x_{1} \frac{\partial}{\partial v_{1}}+\mu x_{2} \frac{\partial}{\partial v_{2}}
$$

for non-vanishing constants $\lambda, \mu$. Without loss of generality, we can always suppose that $\mu=1$. Taking:

$$
X_{1}=v_{1} \frac{\partial}{\partial x_{1}}+v_{2} \frac{\partial}{\partial x_{2}}+f_{1}(\mathbf{x}, \mathbf{v}) \frac{\partial}{\partial v_{1}}+f_{2}(\mathbf{x}, \mathbf{v}) \frac{\partial}{\partial v_{2}}
$$

the vector field $X_{3}=\left[X_{1}, X_{2}\right]$ is given by:

$$
X_{3}=-\lambda x_{1} \frac{\partial}{\partial x_{1}}-x_{2} \frac{\partial}{\partial x_{2}}+\left(\lambda v_{1}-\lambda x_{1} \frac{\partial f_{1}}{\partial v_{1}}-x_{2} \frac{\partial f_{1}}{\partial v_{2}}\right) \frac{\partial}{\partial v_{1}}+\left(v_{2}-\lambda x_{1} \frac{\partial f_{2}}{\partial v_{1}}-x_{2} \frac{\partial f_{2}}{\partial v_{2}}\right) \frac{\partial}{\partial v_{2}}
$$

As the commutator $\left[X_{2}, X_{3}\right]$ does not have nonzero components in $\frac{\partial}{\partial x_{1}}$ and $\frac{\partial}{\partial x_{2}}$, the only possibility is that:

$$
\left[X_{2}, X_{3}\right]=\alpha X_{2}
$$

for some constant $\alpha$. This means that with this realization, the possible Vessiot-Guldberg-Lie algebras are $\mathfrak{r}_{2}, \mathfrak{r}_{4}$ and $\mathfrak{r}_{5}$. Starting from the assumption Equation (42), the components in $\frac{\partial}{\partial v_{1}}$ and $\frac{\partial}{\partial v_{2}}$ imply the constraints:

$$
\begin{array}{r}
2 \lambda^{2} x_{1}-\lambda^{2} x_{1}^{2} \frac{\partial^{2} f_{1}}{\partial v_{1}^{2}}-2 \lambda x_{1} x_{2} \frac{\partial^{2} f_{1}}{\partial v_{1} \partial v_{2}}-x_{2}^{2} \frac{\partial^{2} f_{1}}{\partial v_{2}^{2}}-\alpha \lambda x_{1}=0 \\
2 x_{2}-\lambda^{2} x_{1}^{2} \frac{\partial^{2} f_{2}}{\partial v_{1}^{2}}-2 \lambda x_{1} x_{2} \frac{\partial^{2} f_{2}}{\partial v_{1} \partial v_{2}}-x_{2}^{2} \frac{\partial^{2} f_{2}}{\partial v_{2}^{2}}-\alpha x_{2}=0
\end{array}
$$

Introducing the auxiliary variable $W_{0}=\lambda x_{1} v_{2}-x_{2} v_{1}$, the solution to the system can be written as:

$$
\begin{aligned}
& f_{1}(\mathbf{x}, \mathbf{v})=\frac{v_{1}^{2}}{x_{1}}-\alpha \frac{v_{1}^{2}}{2 \lambda x_{1}}+F_{11}\left(x_{1}, x_{2}, W_{0}\right)+\frac{v_{1}}{x_{1}} F_{12}\left(x_{1}, x_{2}, W_{0}\right) \\
& f_{2}(\mathbf{x}, \mathbf{v})=\frac{1}{\lambda^{2}} \frac{x_{2} v_{1}^{2}}{x_{1}^{2}}-\alpha \frac{x_{2} v_{1}^{2}}{2 \lambda^{2} x_{1}^{2}}+F_{21}\left(x_{1}, x_{2}, W_{0}\right)+\frac{v_{1}}{x_{1}} F_{22}\left(x_{1}, x_{2}, W_{0}\right)
\end{aligned}
$$

With this choice of functions, it follows from the Jacobi identity that the bracket $\left[X_{1}, X_{3}\right]$ must have the following form:

$$
\left[X_{1}, X_{3}\right]=\varepsilon X_{1}+\gamma X_{2}
$$

The term in $X_{2}$ is inessential, so that without loss of generality, we can suppose that $\gamma=0$. Analyzing the components in $\frac{\partial}{\partial x_{1}}$ and $\frac{\partial}{\partial x_{2}}$, we see that the conditions:

$$
F_{12}\left(x_{1}, x_{2}, W_{0}\right)-(\alpha+\varepsilon) v_{1}=0 ; F_{22}\left(x_{1}, x_{2}, W_{0}\right)-(\varepsilon+2) W_{0}+(\alpha+\varepsilon)=0
$$

must be satisfied. This can only happen if $\varepsilon=-\alpha$, from which we conclude that:

$$
F_{12}\left(x_{1}, x_{2}, W_{0}\right)=0, F_{22}\left(x_{1}, x_{2}, W_{0}\right)=(-\alpha+2) W_{0}
$$


With these values, it only remains to evaluate the components in $\frac{\partial}{\partial v_{1}}$ and $\frac{\partial}{\partial v_{2}}$ of $\left[X_{1}, X_{3}\right]$ : in terms of the variables $\left\{x_{1}, x_{2}, W_{0}\right\}$, the conditions read:

$$
\begin{aligned}
& \lambda x_{1} \frac{\partial F_{11}}{\partial x_{1}}+x_{2} \frac{\partial F_{11}}{\partial x_{2}}+(1+\lambda-\alpha) \frac{\partial F_{11}}{\partial W_{0}}+(2 \alpha-\lambda) F_{11}\left(x_{1}, x_{2}, W_{0}\right)=0 \\
& \lambda x_{1} \frac{\partial F_{21}}{\partial x_{1}}+x_{2} \frac{\partial F_{21}}{\partial x_{2}}+(1+\lambda-\alpha) \frac{\partial F_{21}}{\partial W_{0}}+(2 \alpha-1) F_{21}\left(x_{1}, x_{2}, W_{0}\right)=0
\end{aligned}
$$

We thus obtain the functions:

$$
F_{11}\left(x_{1}, x_{2}, W_{0}\right)=\Psi_{1}\left(\frac{x_{2}^{\lambda}}{x_{1}}, \frac{W_{0}^{\lambda}}{x_{1}^{(\lambda+1-\alpha)}}\right) x_{1}^{\left(1-2 \frac{\alpha}{\lambda}\right)} ; F_{21}\left(x_{1}, x_{2}, W_{0}\right)=\Psi_{2}\left(\frac{x_{2}^{\lambda}}{x_{1}}, \frac{W_{0}^{\lambda}}{x_{1}^{(\lambda+1-\alpha)}}\right) x_{1}^{\left(\frac{1-2 \alpha}{\lambda}\right)}
$$

From the $t$-dependent vector field $\mathbf{X}(t, x, v)=X_{1}+F_{2}(t) X_{2}$ and replacing the $v_{i}$ by the first derivatives $\dot{x}_{i}$, we obtain the following SODE Lie system admitting the Lie algebra generated by Equations (40) and (41) as the Vessiot-Guldberg-Lie algebra:

$$
\begin{array}{r}
\ddot{x}_{1}-\left(1-\frac{\alpha}{2 \lambda}\right) \frac{\dot{x}_{1}^{2}}{x_{1}}-\Psi_{1}\left(\frac{x_{2}^{\lambda}}{x_{1}}, \frac{W_{0}^{\lambda}}{x_{1}^{(\lambda+1-\alpha)}}\right) x_{1}^{\left(1-2 \frac{\alpha}{\lambda}\right)}-\lambda F_{2}(t) x_{1}=0 \\
\ddot{x}_{2}+\frac{2-\alpha}{2 \lambda^{2}} \frac{x_{2} \dot{x}_{1}^{2}}{x_{1}^{2}}+\frac{\alpha-2}{\lambda} \frac{\dot{x}_{1} \dot{x}_{2}}{x_{1}}-\Psi_{2}\left(\frac{x_{2}^{\lambda}}{x_{1}}, \frac{W_{0}^{\lambda}}{x_{1}^{(\lambda+1-\alpha)}}\right) x_{1}^{\left(\frac{1-2 \alpha}{\lambda}\right)}-\lambda F_{2}(t) \dot{x}_{2}=0
\end{array}
$$

From the commutators, we see at once that the algebra is isomorphic to $\mathfrak{r}_{2}=\mathfrak{s l}(2, \mathbb{R})$ if $\alpha \neq 0$ and to $\mathfrak{r}_{4}$ if $\alpha=0$. In particular, for the special values $\lambda=1, \alpha=2$ and $F_{2}(t)=-\omega^{2}(t)$, the preceding SODE Lie system reduces to:

$$
\ddot{x}_{1}-\Psi_{1}\left(\frac{x_{2}}{x_{1}}, W_{0}\right) x_{1}^{-3}+\omega^{2}(t) x_{1}=0, \quad \ddot{x}_{2}-\Psi_{2}\left(\frac{x_{2}}{x_{1}}, W_{0}\right) x_{1}^{-3}+\omega^{2}(t) x_{2}=0
$$

corresponding to the well-known generalizations of Ermakov systems (see, e.g., [5,17] and the references therein).

We remark that a slight variation in the preceding argumentation also allows us to obtain SODE Lie systems having the Lie algebra $\mathfrak{r}_{5}$ as the Vessiot-Guldberg-Lie algebra. The system $\mathbf{X}(t, x, v)=$ $X_{1}+F_{2}(t) X_{2}$ in particular has the form:

$$
\begin{array}{r}
\ddot{x}_{1}-\frac{\dot{x}_{1}^{2}}{x_{1}}-x_{1} \Psi_{1}\left(\frac{x_{2}^{\lambda}}{x_{1}}, \frac{W_{0}^{\lambda}}{x_{1}^{(\lambda+1)}}\right)+x_{1} \ln \left(x_{1}\right)-\lambda F_{2}(t) x_{1}=0 \\
\ddot{x}_{2}+\frac{1}{\lambda^{2}} \frac{x_{2} \dot{x}_{1}^{2}}{x_{1}^{2}}-\frac{2}{\lambda} \frac{\dot{x}_{1} \dot{x}_{2}}{x_{1}}-\Psi_{2}\left(\frac{x_{2}^{\lambda}}{x_{1}}, \frac{W_{0}^{\lambda}}{x_{1}^{(\lambda+1)}}\right) x_{1}^{(1-\lambda)}+\frac{x_{2} \ln \left(x_{1}\right)}{\lambda}-\lambda F_{2}(t) \dot{x}_{2}=0
\end{array}
$$

\section{Conclusions}

By means of a direct approach, scalar SODE Lie systems admitting a Vessiot-Guldberg-Lie algebra of a dimension at most three have been constructed. These equations encompass some of the well-known types of SODE Lie systems, like the Liouville-type equations and the Milne-Pinney and Kummer-Schwarz equations. The case of SODE Lie systems in the plane, much more cumbersome due to the complicated partial differential equations arising from the constraints, has been considered for a special case that however contains an important class, namely the generalized Ermakov systems [5].

The problem of determining Vessiot-Guldberg-Lie algebras corresponds formally to studying the realization of Lie algebras in terms of vector fields in $\mathbb{R}^{n}$, and thus, the ansatz could be formulated 
in terms of the primitive classes of realizations $[7,8]$. Such an approach, combined with the lattice of subalgebras of a Lie algebra and the obstructions obtained for low dimensional Vessiot-Guldberg-Lie algebras, could eventually lead to a complete classification of scalar SODE Lie systems. From a pure practical perspective, however, it can be sometimes more convenient to choose a realization that generalizes some fixed realization, with the purpose of obtaining a class of equations or systems that naturally generalize already known types of SODE Lie systems. Proceeding along these lines also enables us to consider realizations depending on parameters, thus enabling the use of non-isomorphic Lie algebras simultaneously. In this context, an interesting problem concerns the combination of the notion of contractions of (realizations of) Lie algebras with that of Vessiot-Guldberg-Lie algebras, especially in connection with the recently developed notion of strong contractions [18].

Acknowledgments: The author acknowledges the referees for useful remarks and suggestions that have greatly improved the presentation. This work was partially supported by the research project MTM2013-43820-P of the Ministerio de Economía y Competitividad (Spain).

Conflicts of Interest: The author declares no conflict of interest.

\section{References}

1. Lie, S. Vorlesungen über Differentialgleichungen mit Bekannten Infinitesimalen Transformationen, B. G. Teubner: Leipzig, Germany, 1891.

2. Cariñena, J.F.; de Lucas, J. Lie systems: Theory, generalisations and applications. Dissertationes Math. 2011, $479,1-162$.

3. Ibragimov, N.K. Integration of systems of first-order equations admitting a nonlinear superposition. J. Nonl. Math. Phys. 2009, 16, 137-147.

4. Ballesteros, A.; Blasco, A.; Herranz, F.J.; de Lucas, J.; Sardón, C. Lie-Hamilton systems on the plane: Theory, classification and applications. J. Diff. Equations 2015, 258, 2873-2907.

5. Cariñena, J.F.; de Lucas, J.; Rañada, M.F. Recent applications of the theory of Lie systems in Ermakov systems. Symmetry Integrability Geom. Methods Appl. 2008, 4, doi:10.3842/SIGMA.2008.031.

6. Cariñena, J.F.; Grabowski, J.; de Lucas, J. Superposition rules for higher-order systems and their applications. J. Phys. A: Math. Theor. 2012, 45, doi:10.1088/1751-8113/45/18/185202.

7. González-López, A.; Kamran, N.; Olver, P.J. Lie algebras of vector fields in the real plane. Proc. Lond. Math. Soc. 1992, 64, 339-368.

8. Shnider, S.; Winternitz, P. Classification of systems of nonlinear ordinary differential equations with superposition principles. J. Math. Phys. 1984, 25, 3155-3165.

9. Ibragimov, N.K.; Gainetdinova A.A. Three-dimensional dynamical systems admitting nonlinear superposition with three-dimensional Vessiot-Guldberg-Lie algebras. Appl. Math. Lett. 2016, 52, 126-131.

10. Cariñena, J.F.; de Lucas, J. Superposition rules and second-order Riccati equations. J. Geom. Mech. 2011, 3, $1-22$.

11. Ibragimov, N.Kh. Group analysis of ordinary differential equations and the invariance principle in mathematical physics. Uspekhi Mat. Nauk 1992, 47, 83-144.

12. Ovsyannikov, L.V. Lectures on the Theory of Group Properties of Differential Equations; Ibragimov, N.K., Ed.; World Scientific: Singapore, 2013.

13. Wei, J.; Norman, E. Lie algebraic solution of linear differential equations. J. Math. Phys. 1963, 4, 575-581.

14. Campoamor-Stursberg, R. Reduction of the symmetry algebra of ODEs by means of additional constraints associated to a subalgebra. Cubo Math. J. 2006, 8, 25-34.

15. Šnobl, L.; Winternitz, P. Classification and Identification of Lie Algebras; American Mathematical Society: Providence, RI, USA, 2014.

16. Lahno, V.I.; Spichak, S.V.; Stognii', V.I. Symmetry Analysis of Evolution Type Equations; Institute of Computer Science: Moscow-Izhevsk, UK, 2004. (In Russian) 
17. Perelomov, A.M. Integrable Systems of Classical Mechanics and Lie Algebras; Birkhäuser Verlag: Basel, Switzerland, 1990.

18. Subag, E.M.; Baruch, E.M.; Birman, J.L.; Mann, A. Strong contractions of the representations of the three dimensional Lie algebras. J. Phys. A: Math. Theor. 2012, 45, doi:10.1088/1751-8113/45/26/265206.

(C) 2016 by the author; licensee MDPI, Basel, Switzerland. This article is an open access article distributed under the terms and conditions of the Creative Commons by Attribution (CC-BY) license (http://creativecommons.org/licenses/by/4.0/). 